\title{
Testing for Placebo Effects Using Data from Blinded, Randomized Controlled Trials
}

\author{
Anup Malani ${ }^{1}$
}

December 2, 2003

\footnotetext{
${ }^{1}$ Anup Malani is Associate Professor, University of Virginia, Charlottesville, VA 22903 (email: amalani@virginia.edu). The author thanks Gary Becker, James Heckman, Feifang Hu, Darius Lakdawalla, Willard Mannng, Ron Michener, Charles Mullin, Tomas Philipson, and Andrew Sellgren; and workshop participants at the University of Virginia, RAND, the University of Southern California, the University of California-Irvine, and the University of Chicago for helpful comments.
} 


\begin{abstract}
This paper proposes a test for the existence of placebo effects, as described by the so-called expectancy theory. This theory, which is the dominant medical theory of how placebo effects operate, posits that health outcomes rise in individuals' beliefs about the probability that they are getting a beneficial treatment and their beliefs about the efficacy of that treatment. Blinded, randomized, controlled trials provide near-perfect environments in which to test this theory because they offer objective, controlled manipulations of subjects' beliefs about treatment. If the expectancy theory is correct, outcomes in trials offering a higher probability of receiving an experimental treatment should be superior to outcomes in trials offering a lower probability of receiving that treatment, conditional on treatment assignment. The paper applies this test to data from over 200 trials of anti-ulcer medications and finds robust evidence of placebo effects in trials of $\mathrm{H}_{2}$-blockers (e.g., Zantac, Tagamet and Pepcid) and of proton-pump inhibitors (e.g., Prilosec, Nexium, and Prevacid). Indeed, trials of $\mathrm{H}_{2}$-blockers manifest placebo effects that are 50 percent as large as the physiological effects of these medications. Because placebo effects are not confined to clinical trials, this result suggests that the standard difference-in-means estimator of treatment effects may seriously underestimate the efficacy of anti-ulcer medications.
\end{abstract}

Keywords: informed consent, self-selection, expected utility, ulcer, generalized least squares. 
Placebo effects can roughly be defined as that component of health outcomes that cannot be attributed to the physiological effects of treatment or to the natural progression of disease. There is a lively debate in the medical literature about whether placebo effects actually exist. On one side is, e.g., a recent New England Journal of Medicine article (Hrobjartsson and Gotzsche, 2001) that examines 114 studies with both a blinded placebo-control group and an unblinded notreatment group and finds few systematic differences in outcomes between these groups. This result does not disprove the existence of placebo effects because it is consistent with the theory that members of unblinded no-treatment groups seek out alternative medication, which elevates their health outcomes.

On the other side of the debate are, e.g., studies (Kirsch and Sapirstein, 1998; Kirsch and Nicholls, 2002) that point to evidence that placebo-control groups in blinded trials often manifest substantially improved health outcomes. These findings are weak support for placebo effects because the improvements could be due to the natural progression of disease. Better studies employ a balanced-placebo design wherein subjects are first randomized across treatments and then across instructions about the value of treatment or whether one obtained placebo. Unfortunately, not only are the results of these studies mixed (Marlatt and Rohsenow, 1980; Kirsch and Weixel, 1988; Penick and Hinkle, 1964; Penick and Fisher, 1965), but their design is ethically questionable if not illegal.

A common weakness of studies on both sides of the debate is that they do not begin with a clear model of placebo effects that can be definitively falsified. This paper addresses this shortcoming by focusing on the dominant medical theory (Jensen and Karoly, 1991) for how placebo effects operate. This so-called expectancy theory posits that health outcomes rise in individuals' beliefs about the probability that they are getting a beneficial treatment and their beliefs about the efficacy of that treatment (Pollo and Benedetti, 2001; Price and Nicholls, 1999). The paper formalizes this theory by assuming that health outcomes are a function not just of the treatment, but also of the expected value of treatment in the eyes of the patient.

The central insight of the paper is that randomized, controlled trials (RCT), if blinded, provide an objective and controlled manipulation of beliefs that permits a relatively clean test of the expectancy theory of placebo effects. Informed consent reveals to subjects the ex ante probability of obtaining treatment, but blinding ensures that they do not learn their ultimate assignment. This 
probability of treatment, along with subjects' assessments of the relative efficacy of the treatment, affects subjects' beliefs about the expected value of the trial. If the expectancy theory is correct, then this probability also affects outcomes. More specifically, because enrollment in trials is voluntary, trials only attract individuals who believe the treatment is better than the control. If there exist placebo effects, trials with a higher probability of treatment should produce better outcomes, conditional on treatment assignment, than trials with a lower probability of treatment.

The paper applies this test for placebo effects to data from over 200 RCTs of anti-ulcer medications. The advantage of ulcer trials is that outcomes are objectively measured: ulcer healing is verified by endoscopy. In trials where patients were asked for informed consent and thus had some indication of their probability of treatment, a significant, positive correlation is found between this probability and outcomes in the treatment arms of trials of $\mathrm{H}_{2}$-blockers (e.g., Zantac and Tagamet) and proton-pump inhibitors (e.g., Nexium and Prevacid). In trials without informed consent, this correlation is diminished. Control arms also manifest evidence of significant, though weaker placebo effects.

Section 1 presents a model of how individuals sort into trials. Section 2 formalizes the expectancy theory of placebo effects. Section 3 derives testable predictions regarding trial outcomes with and without placebo effects. Section 4 tests these predictions against data from ulcer trials. The appendix offers proofs for the propositions in the text.

\section{TREATMENT STRATEGIES}

This section presents a model of how individuals who are currently ill sort among treatment strategies. Suppose there are two possible, future health states: continued illness $\bar{y}$ and recovery $\underline{y}$, where

$\bar{y}>\underline{y}$. (The analysis can easily be extended to the case of continuous health variables.) Treatments are lotteries over these two states. For now, assume that there exist only two treatments: no treatment $(k=0)$ and an experimental treatment $(k=1)$. Let $y_{k i}$ be the random variable that describes individual $i$ 's health outcome given treatment $k$. Define $p_{k i}=\operatorname{Pr}\left\{y_{k i}=\bar{y} \mid\right.$ no placebo effects $\}$. In the case of no treatment, this probability is simply a function of the natural progression of disease. For the experimental treatment, this probability is also a function of the physiological effects of treatment. Although a slight abuse of medical terminology, the sum of natural progression and 
the physiological effects of treatment will be called the specific effect of treatment.

Treatments are to be distinguished from treatment strategies. The latter are defined to be lotteries over treatments, and thus compound lotteries over health states. Initially assume that there are only two feasible treatment strategies: certain consumption of no treatment $(s=0)$ or enrollment in a randomized, placebo-controlled trial $(\mathrm{RPCT})$ that is blinded $(s=B T)$. The latter strategy entails a probability $d$ of receiving the experimental treatment and probability $1-d$ of receiving a placebo. This narrow set of feasible strategies is appropriate under two conditions. First, the experimental treatment is not available outside the context of the trial because, e.g., the government has not approved it. Second, individuals are only offered one lottery and in this lottery subjects do not learn which treatment they actually consume. This condition is reasonable because trials are costly to conduct and investigators prefer blinded trials due to concerns about attrition.

A common feature of trials important to the analysis in this paper is that enrollment is voluntary and subject to informed consent. As part of this disclosure, it is typically required that subject be given information about the probability that they will receive the experimental treatment. See, e.g., 21 C.F.R. $\S \S 7.3(\mathrm{f}), 20.25$ (United States). Individual $i$ 's belief about the probability that she is consuming treatment $k$ is $\delta_{k i}$. For simplicity, assume that subjects do not consume more than one treatment, so $\sum_{k=0}^{1} \delta_{k i}=1$. From the individual's perspective, strategies are defined by the vector of beliefs $\boldsymbol{\delta}_{i}=\left(\delta_{0 i}, \delta_{1 i}\right)$. A strategy of certain consumption of no treatment is defined by $\boldsymbol{\delta}_{i}=(1,0)$. The trial strategy reveals the probability of treatment but not ultimate treatment assignment, so $\boldsymbol{\delta}_{i}=(1-d, d)$. In order to abstract from problems with attrition, assume that there is no unblinding due to, e.g., subject sampling (Philipson and DeSimone, 1997) on outcomes. The subject's belief about the specific effect of treatment $k$ is $\pi_{k i}$. (No position need be taken on how beliefs about specific effects are formed. Different tests will be proposed for different assumptions about the relationship between efficacy and beliefs.)

Individuals are assumed to have preferences that conform to Savage's axioms (Savage, 1954) and thus permit representation in the form of a subjective expected utility function. Individuals draw utility from health and non-health consumption. For simplicity, assume further that individuals have identical and additively separable utility functions. Let $u(y)$ be the utility from health outcome $y$, with $u^{\prime}>0, u^{\prime \prime}<0$. The expected utility of strategy $s$ to individual $i$ is a weighted 
sum of the utility from each health outcome, with the weights being her subjective beliefs about the probability of each outcome given the compound lottery $s: U_{i}^{s}=\pi_{i}^{s} u(\bar{y})+\left(1-\pi_{i}^{s}\right) u(\underline{y})$. Given that an individual knows her treatment status outside the context of a trial, her subjective belief is $\pi_{i}^{0}=\pi_{0 i}$. Belief about the probability of recovery given a blinded trial strategy depends on the

probability of being given the experimental treatment: $\pi_{i}^{B T}=d \pi_{1 i}+(1-d) \pi_{0 i}$. Each individual is assumed to sort into that strategy which maximizes her utility. (Sorting based on expected utility is not a necessary condition for the tests of placebo effects set forth in the next section. All that is required is a rational actor model that permits sorting based on beliefs about the specific effects of strategies. An advantage of expected utility theory is that it permits this model of sorting into treatment strategies conveniently to be generalized to take into account, e.g., difference in costs across strategies or attrition based on self-sampling if necessary.)

In order to determine the sorting of individuals to strategies, one must know the distribution of beliefs about treatment among the population. Since the object of these beliefs is the actual efficacy of treatment, let $\mathbf{g}_{p}$ give the probability distribution function of $\mathbf{p}_{i}=\left(p_{0 i}, p_{1 i}\right)$ across the population. Let $\mathbf{g}_{\pi}$ give the probability distribution for $\boldsymbol{\pi}_{i}=\left(\pi_{0 i}, \pi_{1 i}\right)$. All distributions discussed are assumed to be well-defined. The expectations operator $E_{g}(\cdot)$ will be employed when expectations are taken over the joint distribution of $\left(\mathbf{p}_{i}, \boldsymbol{\pi}_{i}\right)$.

\section{EXPECTANCY THEORY OF PLACEBO EFFECTS}

According to the expectancy theory of placebo effects, patients manifest changed health outcomes in response to expectations regarding treatment. In particular, the more effective a patient expects a treatment to be, the better her response to it. The more likely a patient thinks she is to get a beneficial treatment the better is her health outcome holding constant whether or not she receives treatment. This section formalizes this theory with a simple model of health outcomes as a weighted average of the specific effects of one's treatment and beliefs about the specific effects of one's treatment strategy:

$$
\operatorname{Pr}\left\{y_{k i}=\bar{y} \mid a_{k}, p_{k i}, \pi_{i}^{s}\right\}=\left(1-a_{k}\right) p_{k i}+a_{k} \pi_{i}^{s},
$$


where $a_{k} \in[0,1]$ for all $k$. The relevant beliefs are not those about treatment $k$ but about strategy $s$ because, if an individual enters a blinded trial, she does not learn whether she has consumed treatment $k$. The parameter $a_{k}$ indicates the relative importance of beliefs in determining the health outcome given consumption of treatment $k$. Placebo effects are defined to exist for treatment $k$ if $a_{k}>0$. The influence of beliefs is assumed not to vary across individuals. The purpose of this assumption is to facilitate application of the test for the existence of placebo effects in section 3 to ulcer trial data, which are aggregated to the level of treatment groups so estimation of a random effects model is not feasible. An implication of this assumption is that the influence of placebo effects is independent of individuals' beliefs about the efficacy of different treatments. There is no compelling medical reason to think otherwise.

\section{TESTS FOR EXISTENCE OF PLACEBO EFFECTS}

This section employs the models of sections 1 and 2 to generate predictions regarding outcomes observed in clinical trials with and without placebo effects. Any difference in predictions can be used to test for the existence of placebo effects. The results are summarized in table 1.

Initially, assume that only individuals who believe that the experimental treatment is superior to no treatment $\left(\pi_{1 i}>\pi_{0 i}\right)$ sort into the trial. (The next section will justify this assumption.) Define $\tilde{\pi}_{k i}=\pi_{k i}-\pi_{0 i}$, the relative benefit of treatment $k$ over no treatment, so that $\pi_{1 i}>\pi_{0 i}$ can be written $\tilde{\pi}_{1 i}>0$. In the absence of placebo effects, the mean outcome observed in the group that receives treatment $k$ in a blinded RPCT is $E_{g}\left[y_{k} \mid \tilde{\pi}_{1}>0\right]=E_{g}\left[p_{k} \mid \tilde{\pi}_{1}>0\right]$. (Henceforth, $i$ subscripts are dropped to simplify notation whenever their use does not add to the exposition.) In the presence of placebo effects, the mean outcome is

$$
E_{g}\left[y_{k} \mid \tilde{\pi}_{1}>0, d\right]=\left(1-a_{k}\right) E_{g}\left[p_{k} \mid \tilde{\pi}_{1}>0\right]+a_{k}\left\{d E_{g}\left[\pi_{1} \mid \tilde{\pi}_{1}>0\right]+(1-d) E_{g}\left[\pi_{0} \mid \tilde{\pi}_{1}>0\right]\right\} .
$$

Without placebo effects, outcomes are solely a function of the specific effects of treatment. The probability of treatment or, equivalently, the share treated is irrelevant. With placebo effects, outcomes are also a function of beliefs about specific effects of the treatment strategy. Because these beliefs depends on the probability of receiving the experimental treatment, i.e., $\pi_{i}^{B T}=d \pi_{1 i}+$ 
$(1-d) \pi_{0 i}$, so too will outcomes. More specifically, an increase in the share treated lifts mean outcomes in each group of the trial: $\partial E_{g}\left[y_{k} \mid \tilde{\pi}_{1}>0, d\right] / \partial d=a E_{g}\left[\tilde{\pi}_{1} \mid \tilde{\pi}_{1}>0\right]>0$. As the share treated rises, individuals' expectations of the trial rise because there is a better chance of getting the experimental treatment, which is thought to be better than no treatment. These expectations translate into better average outcomes when there exist placebo effects. This yields the following test for placebo effects:

Proposition 1 Suppose individuals enroll in a blinded RPCT if and only if $\tilde{\pi}_{1 i}>0$. If trials that have higher treatment shares but are otherwise identical yield higher mean outcomes conditional on treatment, then there exist placebo effects.

This is the central theoretical result of the paper. It is robust to the functional form of health outcomes.

\subsection{Self-Selection}

This section justifies the assumption in proposition 1 that individuals enroll in a blinded RPCT if and only if they believe that the specific effects of the experimental treatment are superior to those of no treatment. This sorting is a direct implication of individual self-selection into the type of trial described in section 1 . In that context, expected utility maximization implies that individual $i$ will enroll in a trial if and only if the probability of recovery given enrollment in a blinded RCT is greater than the probability of recovery given no treatment. If subjects do not take placebo effects into account when deciding whether to enroll, this condition can be written $\pi_{i}^{B T}=d \pi_{1 i}+(1-d) \pi_{0 i}>\pi_{0 i}$ and is satisfied if and only if $\tilde{\pi}_{1 i}>0$. This condition does not depend on the share treated. This result yields the following conclusion.

Proposition 2 Given the model of treatment strategies and preferences in section 1, self-selection implies that individuals will enroll in a blinded RPCT if and only if $\tilde{\pi}_{1 i}>0$. Therefore, the test for placebo effects in proposition 1 is valid for this model.

The test remains valid even if subjects take placebo effects into account when deciding whether to enroll in trials. (An expected utility representation for preferences is feasible in this case if beliefs about the specific effects of treatments are treated as state variables.) The individual assesses the 
value of the blinded trial to be $\pi_{i}^{B T}=d\left[\left(1-\alpha_{1 i}\right) \pi_{1 i}+\alpha_{1 i} \pi_{i}^{B T}\right]+(1-d)\left[\left(1-\alpha_{0 i}\right) \pi_{0 i}+\alpha_{0 i} \pi_{i}^{B T}\right]$, where $\alpha_{k i}$ is the individual's belief regarding the influence $a_{k}$ of beliefs on outcomes. Implicit in this formulation is the assumption that beliefs about the influence of beliefs are independent of beliefs about the specific effects of treatments. A little algebra reveals that the condition $\pi_{i}^{B T}>\pi_{0 i}$ again collapses to $\tilde{\pi}_{1 i}>0$. Proposition 2 is not valid, however, if investigators offer individuals incentives such as cash or in-kind benefits to participate in a trial. Such payments may induce individuals who believe that no treatment is superior to experimental treatment to enroll. An increase in the share treated lowers the expectations and thus outcomes of these individuals. The overall effect of an increase in the share treated will be ambiguous.

\subsection{Conventional Treatment}

Thus far it has been assumed that there are only two treatments and two strategies. If individuals have available a third treatment and strategy, namely conventional treatment outside the context of a trial, there is the possibility of self-selection based on the share treated. If there is also a correlation between individual beliefs — which drive sorting — and outcomes, then it is not obvious that the test for placebo effects set forth in proposition 1 will work. Without placebo effects, the share treated may affect outcomes through self-selection. With placebo effects, share treated will affect outcomes both due to a direct relationship between beliefs and outcomes and due to self-selection. In the presence of a conventional alternative, self-selection is in effect noise that may obscure identification of any direct relationship between beliefs and outcomes. One solution is to rely on the fact that placebo effects imply a positive relationship between share treated and outcomes and search for conditions under which self-selection implies a negative relationship between share treated and outcomes. Under these conditions, one could continue to test for placebo

effects by searching for a positive relationship between share treated and outcomes. This section sets forth these conditions, which, it turns out, are fairly reasonable.

To see that the presence of a conventional treatment implies self-selection based on share treated, let $k=2$ indicate the conventional treatment. An individual will now enroll in a blinded RPCT if and only if $d \pi_{1 i}+(1-d) \pi_{0 i}>\max \left\{\pi_{0 i}, \pi_{2 i}\right\}$ or, equivalently,

$$
\tilde{\pi}_{1 i}>\max \left\{0, \tilde{\pi}_{2 i} / d\right\}
$$


In words, an individual will enroll in a trial so long as the expected value of its lottery over the the experimental treatment and no treatment is greater than the value of no treatment or conventional treatment for sure. Based on the subject's beliefs, if no treatment is superior to the conventional alternative, she will enroll if an only if the experimental treatment is better than no treatment - the same selection condition as in the case without a conventional treatment. If, however, the conventional treatment is better than no treatment, but worse than the experimental treatment, the enrollment decision will depend on the share treated. If along the continuum from the experimental treatment to no treatment, the conventional treatment is closer to the experimental treatment, it will take a high probability of obtaining the experimental treatment to attract an individual to the trial. If the conventional treatment is closer in efficacy to no treatment, then even a small probability of obtaining the experimental treatment may attract the individual to the trial.

Examining solely the subpopulation for which the share treated affects the enrollment decision, the following proposition gives conditions under which an increase in the share treated reduces average beliefs about the specific effects of the experimental treatment among the members of the subpopulation that enroll.

Proposition 3 Suppose that $E_{g}\left[\tilde{\pi}_{k i} \mid \pi_{0 i}\right]=E_{g}\left[\tilde{\pi}_{k i}\right]$; that $\tilde{\pi}_{k i}>0$ for $k=1,2$; and that $\left(\ln \tilde{\pi}_{1 i}, \ln \tilde{\pi}_{2 i}\right)$ have a non-degenerate log-concave or log-convex joint density with mean $\left(\mu_{1}, \mu_{2}\right)$ and variance $\Sigma$. Define $u_{k i}=\ln \tilde{\pi}_{k i}-\mu_{i}, W_{i}=u_{1 i}-u_{2 i}, \sigma=\sigma_{11}+\sigma_{22}-2 \sigma_{12}, b_{1}=\left(\sigma_{11}-\sigma_{12}\right) / \sigma, b_{2}=a_{1}-1$, and $V_{i}=b_{1} u_{2 i}-b_{2} u_{1 i}$. By construction $u_{i}=b_{i} W_{i}+V_{i}$, where $W_{i}$ and $V_{i}$ are uncorrelated. Suppose further that $W_{i}$ and $V_{i}$ are actually independent. Define $\rho_{12}=\operatorname{corr}\left(\ln \tilde{\pi}_{1 i}, \ln \tilde{\pi}_{2 i}\right)$. If $\sigma_{11}>\sigma_{12}$ or, equivalently, $\rho_{12}<\sigma_{1} / \sigma_{2}$, then $\partial E_{g}\left[\pi_{1 i} \mid \tilde{\pi}_{1 i}>\tilde{\pi}_{2 i} / d\right] / \partial d \leq 0$ and $\partial E_{g}\left[\pi_{0 i} \mid \tilde{\pi}_{1 i}>\tilde{\pi}_{2 i} / d\right] / \partial d=0$.

Note three things. First, one can write $\pi_{k i}=\pi_{0 i}+\tilde{\pi}_{k i}$, for $k=1,2$. Because natural progression is defined as the probability of recovery without treatment, this equation says that $\tilde{\pi}_{k i}$ is individual $i$ 's beliefs about the physiological effects of treatment $k$. Therefore, the assumption that $\tilde{\pi}_{k i}$ and $\pi_{0 i}$ are independent implies that the physiological effects of $k$ are independent of the natural progression of disease. This assumption ensures that selection pressures due to changes in treatment share do not affect the distribution of beliefs about no treatment among enrollees. Second, the assumption that $\left(\ln \tilde{\pi}_{1 i}, \ln \tilde{\pi}_{2 i}\right)$ is $\log$-concave or convex is not very restrictive. The class of log-concave densities is itself quite large. It includes, e.g., the bivariate normal distribution. 
In that case, the fact that $W_{i}$ and $V_{i}$ are (by construction) uncorrelated implies they are also independent. Finally, proposition 3 is robust to the use of exclusion and inclusion criteria when selecting enrollees so long as these criteria are independent of beliefs about treatment efficacy. The prediction is also robust to imperfect informed consent so long as whether candidates are informed of $d$ via informed consent is independent of the value of $d$.

Proposition 3 says that, so long as the covariance between $(\log )$ beliefs about the physiological effects of the experimental and conventional treatments is less than the variance of (log) beliefs about the experimental treatment, changes in the share treated, if anything, reduce enrollees' expectations regarding the experimental treatment. From the definition of the correlation coefficient, it is obvious that the condition on the covariance is satisfied whenever the variance of beliefs about the physiological effects of the experimental treatment is greater than the variance of beliefs about the conventional treatment. In this light, the condition on the covariance does not appear at all unreasonable. The experimental treatment, by virtue of being new, will be associated with greater uncertainty in beliefs among the patient population than the conventional treatment.

The intuition behind proposition 3 begins with the observation that the trial only attracts individuals who believe that the experimental treatment is so much better than conventional treatment that, even with the risk of obtaining no treatment at all, enrolling in the trial is a superior strategy to conventional treatment. If one alters a trial to increase the probability of obtaining the experimental treatment, a patient who is marginally not optimistic (or marginally too pessimistic about no treatment) enough about the experimental treatment to have risked randomization into the placebo-control group before may now be willing to take that risk because it is smaller.

This logic is valid only if individuals who are optimistic about the experimental treatment aren't too optimistic about conventional treatment as well. If individuals who are more optimistic about the experimental treatment are also (sufficiently) more optimistic about conventional treatment, individuals who are more optimistic about the experimental treatment are not more likely to join a trial at any given level of share treated. Although the value of trial is higher given these individuals' optimism about the experimental treatment, so is their optimism about the conventional alternative. If these individuals are sufficiently optimistic about the alternative, they may prefer it to enrollment in the trial despite their high expectations for the experimental treatment.

Self-selection implies that trials with different treatment shares may have enrollee populations 
with different beliefs about each treatment. If there is no correlation between individual beliefs and individual outcomes, share treated will not affect outcomes in the absence of placebo effects. Although share treated may affect outcomes in the presence of placebo effects, the direction of the relationship is not obvious. Nevertheless, one can test for placebo effect by checking for any sort of correlation between treatment shares and outcomes.

If, however, individual beliefs and individual outcomes are correlated, treatment shares will affect outcomes even in the absence of placebo effects. It is reasonable to suppose this correlation is positive. If it were negative, that would imply individuals who respond to well to treatment estimate that they do not, and those that do not respond well estimate that they do. Although one might suppose individuals over- or under-estimate treatment response, surely individuals do not guess their personal response in the manner suggested by a negative correlation. Therefore, it is assumed that treatment efficacy and beliefs about efficacy are related according to the function

$$
p_{k i}=f\left(\pi_{k i}\right)+v_{k i}
$$

where $f$ is everywhere continuously differentiable, $f^{\prime}>0$, and $v_{k i}$ is independent of $\pi_{k^{\prime} i^{\prime}}$ for all $\left(k^{\prime}, i^{\prime}\right)$ and of $v_{k^{\prime} i^{\prime}}$ for all $\left(k^{\prime}, i^{\prime}\right)$ except $\left(k^{\prime}=k, i^{\prime}=i\right)$. The error term $v_{k i}$ reflects error in predictions of specific efficacy by individual $i$. This assumption permits the following proposition.

Proposition 4 Suppose $\partial E_{g}\left[\pi_{1 i} \mid \tilde{\pi}_{1 i}>\tilde{\pi}_{2 i} / d\right] / \partial d \leq 0$ but $\partial E_{g}\left[\pi_{0 i} \mid \tilde{\pi}_{1 i}>\tilde{\pi}_{2 i} / d\right] / \partial d=0$. If trials that have higher treatment shares but otherwise are identical yield higher mean outcomes in the experimental treatment groups or yield different mean outcomes in no treatment groups, then there exist placebo effects.

The change in trial outcomes due to a change in share treated is a weighted sum of the change in the specific effects of the assigned treatment given self-selection, the change in beliefs about the specific effects of the trial strategy given self-selection, and the change in beliefs about the specific effects of the trial strategy holding self-selection constant:

$$
\frac{\partial E_{g}\left[y_{k} \mid s=B T\right]}{\partial d}=\left(1-a_{k}\right) \frac{\partial E_{g}\left[p_{k} \mid s=B T\right]}{\partial d}+a_{k} \frac{\partial E_{g}\left[\pi^{B T} \mid s=B T\right]}{\partial d}+a_{k} \tilde{\pi}_{1}
$$

The last term is simply the physiological effects of the experimental treatment, which sorting 
implies is positive regardless of the share treated. The second term in (4) is non-zero only in the presence of a conventional-treatment option. Proposition 3 gives the conditions under which an increase in the share treated lowers average beliefs about the specific effects of the experimental treatment and leaves unaltered average beliefs about the specific effects of no treatment. This implies that the change in beliefs about the specific effects of the trial strategy is negative in both the experimental-treatment and the no-treatment groups. The first term in (4) is non-zero only if the specific effects of the assigned treatment and beliefs about those specific effects are correlated - where the direction of causation runs from specific effects to beliefs because subjects form beliefs to predict specific effects. If the correlation is positive, proposition 3 implies that the first term is negative in the experimental treatment group and zero in the no treatment group.

If there are no placebo effects, there is no weight $\left(a_{k}=0\right)$ assigned to the second and third terms in (4), so changes in specific effects are all that drive outcomes. An increase in the share treated ought to lower outcomes in the experimental-treatment group given self-selection. There should be no change in the no-treatment group. If there are placebo effects $\left(a_{k}>0\right)$, then the last two terms in (4) have positive weight. This adds a negative and positive term to the change in outcomes in both groups. According to proposition 4, since outcomes in the experimental-treatment group fall with the share treated even without placebo effects, the presence of placebo effects can only be confirmed if outcomes in this group rise with the share treated. (Of course this implies that the test for the experimental group is prone to false negatives. It is possible that placebo effects exist but that self-selection, which tends to lower outcomes, overwhelms it.) In contrast, since outcomes in the no-treatment group should be unaffected by changes in the share treated without placebo effects, the presence of placebo effects can be confirmed if outcomes in this group respond at all to a change in the share treated.

The results from this section apply to conventional control trials. All that is required is that one switch the subscripts $k=0$ and $k=2$. The results can also be extended to the case where individuals take placebo effects into account when choosing treatment strategies. The selection equation will be $\tilde{\pi}_{1 i}>\max \left\{0, h(d) \tilde{\pi}_{2 i}\right\}$, where $h(d)=\left[d \tilde{\alpha}_{1 i}+(1-d) \tilde{\alpha}_{0 i}\right] / d \tilde{\alpha}_{1 i}$ and $\tilde{\alpha}_{k i}=1-\alpha_{k i}$. Again, the population of enrollees will depend on the share treated. If the specific effects of a treatment and beliefs about those specific effects are uncorrelated, any change in outcomes with the share treated is evidence of placebo effects. If specific effects and beliefs are positively correlated, 
then any test for placebo effects will depend on the nature of self-selection. Because $h^{\prime}(d)<0$ for $d>0.5$, propositions 3 and 4 are valid for trials where more than half of enrollees are treated.

\section{APPLICATION TO ULCER TRIALS}

Of the handful of studies that claim to find evidence to support the expectancy theory of placebo effects, Skovlund (1991) and Pollo and Benedetti (2001) are likely the best. The former found that, conditional on assignment to the treatment or control group, subjects in a placebo-controlled trial of the painkiller paracetamol manifested better outcomes than those in a conventional controlled trial of the same drug. The latter gave post-operative subjects a saline drip; randomized subjects across three instructions about the drip: no comment, the drip is a potent painkiller or placebo, and the drip is a potent painkiller; and separately offered subjects the painkiller buprenorphine on demand. The first instruction induced the greatest demand for buprenorphine and the third the least. While these studies are insightful, they are not compelling support for the expectancy theory. They have small sample sizes and examine subjectively-measured outcomes, namely patient self-reports of pain levels.

This paper addresses these shortcoming by examining nearly 200 trials of anti-ulcer medications, which permit an objective measure of outcomes. Ulcers are the erosion of the mucous lining in the stomach or small intestine and are judged healed only via endoscopy by the investigator. Three types of medication are considered. The first type, $\mathrm{H}_{2}$-blockers, was introduced in 1977. The most popular brands are Tagamet (cimetidine), Zantac (ranitidine), and Pepcid (famotidine). $\mathrm{H}_{2}$-blockers prevent the production of acid in the stomach. The second type of medication, prostaglandins, was introduced in 1987. The most common prostaglandins are misoprostil and enprostil. These drugs build up and thus repair the mucous lining of the stomach and intestine. The third class, proton-pump inhibitors (PPIs), and were introduced after prostaglandins. The most popular brands are Prilosec (omeprazole), Nexium (esomeprazole) and Prevacid (lansoprazole). Like $\mathrm{H}_{2}$-blockers, these medications prevent the production of acid in the stomach. A distinguishing feature of these medications is that they offer a much higher chance of healing an ulcer than do antacids, which are alkali that absorb acid in the stomach.

That being said, it is now recognized that $90 \%$ of non-gastric ulcers are caused by the bacteria 
heliobacter pylori. These infections are usually treated with a combination of antibiotics and $\mathrm{H}_{2^{-}}$ blockers or PPIs. This paper tests for placebo effects in trials where $\mathrm{H}_{2}$-blockers, prostaglandins or PPIs are used in isolation. These trials typically precede the change to antibiotic-based treatments.

\subsection{Data}

The data set (available from the author) includes the published results from over 200 clinical trials studying treatment for pre-pyloric, pyloric and duodenal ulcers. Each of the trials is randomized, parallel-armed, and double-blind, and employs either a placebo, antacid, bismuth subcitrate or conventional control. If conventional controls are employed, they are from either the same or a previous class of medication as the experimental treatment. Importantly, subjects in 197 of the trials were asked for informed consent prior to enrollment. Hence it is reasonable to suppose that subjects in those trials had some indication of their chance of obtaining the experimental treatment.

Data were gathered on the characteristics of trials and of subjects. Data on subjects are aggregated to the arm- or group-level. For example, there are data on the average age of subjects assigned to any given treatment group, but not the age of each subject assigned to that group. Although there are data on subjects in a group as of the date that they are randomized into the group, precise information on how the group changes due to attrition are not available. This omission is handled in three ways, by assuming those who attrite out heal at the same rate as those who remain (method 1$)$, all heal (method 2$)$, or all do not heal (method 3$)$. Table 2 provides summary statistics for the data. Each observation represents a measurement on the indicated arm of the indicated type of trial. Means and standard deviations are calculated weighting each arm in proportion to the number of subjects evaluated per protocol, regardless of the number of measurements on the arm. Frequency of medication and total dosage are not provided for control arms because such variables are meaningless for placebo arms.

There are several things to note about the data. First, there are more groups given the experimental treatment than given the control. The reason is that each trial typically involves one control arm but multiple experimental treatment arms. Typically these arms will vary the total daily dosage or the daily frequency of medication. While individuals may have different beliefs about efficacy of each treatment v. no treatment, it is assumed that individuals do not have refined 
beliefs about efficacy by dosage. Second, the probability of active treatment is estimated by one minus the probability of randomization into the placebo, antacid or bismuth subcitrate group, or a lower-class ulcer medication. PPIs are the highest class and $\mathrm{H}_{2}$-blockers the lowest class drug. Trials with same-class controls are assumed to have a probability of treatment equal to one. Third, the antacid-permitted variable is coded from 1 to 5 . One indicates that subjects were prohibited from taking antacids, two that subjects were discouraged from taking antacids, three that subjects were permitted to take antacids (or the study did not counsel subjects on antacids), four that antacids were provided, and five that antacids were required.

\subsection{Empirical Model}

Assume that the specific effects of treatment $k$ on individual $i$ enrolled in trial $j$ are a deterministic, linear function of the vector $x_{i j}$, which includes a constant, clinical and demographic variables on individual $i$, and structural features of trial $j: p_{k i j}=\beta_{k}^{\prime} x_{i j}$. This is a strong assumption, but because the ulcer trial data are rather coarse there is little benefit from a more nimble parameterization of $p_{k i j}$. Assume that beliefs regarding specific effects are given by $\pi_{k i j}=\gamma_{k}^{\prime} x_{i j}+\varepsilon_{k i j}$, where $\varepsilon_{k i j}$ is independent of $x_{i j}$ and is i.i.d. mean-zero across individuals, trials, and treatment states, with mean zero and variance $\sigma_{\varepsilon}$. The condition on $\varepsilon_{k i j}$ implies that individual errors in predicting specific treatment response do not depend on the treatment. If $\gamma_{k}=\beta_{k}$, then this parameterization of beliefs implies rational expectations.

Because trials often take multiple measurements on each individual, a treatment's effect is cast as a hazard rate. Assuming it is constant over time, (1) and the assumed parameterization of $\left(p_{k i j}, \pi_{i j}\right)$ imply $-\ln S_{i j k}(t) / t=\theta_{(x) k} x_{i j}+\theta_{(x d) k} d_{j} x_{i j}+\eta_{i j k}$, where $S_{i j k}(t)$ gives the probability of still having an unhealed ulcer on date $t$, the right-hand side is the hazard rate into healing, $\theta_{(x) k}=\left(1-a_{k}\right) \beta_{k}+a_{k} \gamma_{0}, \theta_{(x d) k}=a_{k}\left(\gamma_{1}-\gamma_{0}\right)$, and $\eta_{i j k}=a_{k} d_{j} \varepsilon_{1 i j}+a_{k}\left(1-d_{j}\right) \varepsilon_{0 i j}$. Summing over individuals and dividing by $n_{j k}$, the number of subjects enrolled in treatment arm $k$ of trial $j$, yields the regression equation $-\left\langle\ln S_{j k}(t)\right\rangle / t=\theta_{(x) k} \bar{x}_{j k}+\theta_{(x d) k} d_{j} \bar{x}_{j k}+\bar{\eta}_{j k}$. The left-hand side is approximated, first, by a first-order Taylor approximation around $\bar{S}_{j k}(t)$, the average probability of remaining ill at $t$, which in turn the observed group survival rate. Approximating $\bar{S}_{j k}(t)$ is difficult because data on subjects who attrite out of the trials are not available. Therefore, $\bar{S}_{j k}(t)$ is approximated with $\bar{y}_{j k t}$ under three different assumptions about the healing rate of those who 
attrite (methods 1 - 3 referenced earlier).

The regression equation that is ultimately estimated is

$$
-\ln \left(\bar{y}_{j k t}\right) / t=\theta_{(x) k} \bar{x}_{j k}+\theta_{(x d) k} d_{j} \bar{x}_{j k}+\omega_{j k}
$$

where the unit of time is one day and $\omega_{j k}=\bar{\eta}_{j k}+u_{j k t}+v_{j k t}$. The error term $u_{j k t}$ is from approximating $\bar{S}_{j k}(t)$ with $\bar{y}_{j k t}$. The Lagrange remainder from approximating $\left\langle\ln S_{j k}(t)\right\rangle$ with $\ln \bar{S}_{j k}(t)$ is absorbed into the coefficient on the constant. For simplicity this is left out of the definition of $\theta_{(x) k}$. The error term $v_{j k t}$ captures the variation in the remainder across arms and trials.

Because the relevant hazard rate, $\operatorname{Pr}\left\{y_{k i}=\bar{y} \mid s\right\}$, is confined by assumption to $[0,1]$, the empirical model requires estimation of a linear probability model. While that model has flaws, it is not wholly inappropriate for the application in this paper. As a theoretical matter, the dependent variable in (5) can range from $(0, \infty)$. Moreover, because individuals in an arm are aggregated, the error term is more likely to resemble a normal distribution.

In the presence of a conventional alternative, the tests for placebo effects set forth in section 3 may be complicated by self-selection based on share treated, which implies that $E\left(\varepsilon_{k i j} d_{j} \mid s=B T\right) \neq$ 0. This problem is addressed by including trial- and subject-level covariates that may capture selection. An alternative approach is to isolate a subsample of trials for which there is no conventional alternative to the experimental treatment. This is only possible with $\mathrm{H}_{2}$-blocker trials before 1987 , when prostaglandins were introduced. For each of the other classes of drugs, $\mathrm{H}_{2}$-blockers are a conventional alternative. A third approach to selection is to assume $E\left(\varepsilon_{k i j} d_{j} \mid s=B T\right)=0$ but partition $\bar{x}_{j}=\left(\bar{x}_{j}^{o}, \bar{x}_{j}^{u}\right)$, where $\bar{x}_{j}^{o}$ is observable but $\bar{x}_{j}^{u}$ may not be. Suppose that $\bar{x}_{j}^{o}$ is chosen such that, as a theoretical matter, it ought to be $\theta_{(x d) k}^{o} d_{j} \bar{x}_{j}^{1}>0$ and that, given that selection pressures depend on $d_{j}, \theta_{(x d) k}^{u} d_{j} \bar{x}_{j}^{u}=\phi_{(x d) k} d_{j}^{2}+e_{j k}$, where $e_{j k}$ is independent of $d_{j}$ and $\bar{x}_{j}^{o}$. If selection is a problem but there are no placebo effects, $\theta_{(x d) k}^{u}$ and thus $\phi_{(x d) k}$ should be zero. If the estimate of $\phi_{(x d) k}$ is significantly different from zero, then there must exist placebo effects or selection is not a problem. These alternative approaches yield, if anything, stronger evidence of placebo effects than when selection is controlled with appropriate covariates.

It should be noted that (5) was modified and estimated as a generalized linear model with a 
log link and survival distributed binomial or gamma. The results are similar, but nearly every covariate specification performs worse on Pregibon's (Pregibon, 1980) link test when survival is assumed gamma. In addition, a proportional hazard model was also estimated and produced somewhat stronger evidence of placebo effects. The coefficient on time is positive and significant, suggesting the risk of healing rises over time. However, few covariate specifications pass the link test.

\subsection{Results}

Table 3 presents results for $\mathrm{H}_{2}$-blocker trials. Estimation was by feasible GLS. Observations were weighted such that each arm makes a contribution to estimates in proportion to the number of subjects in the arm, regardless of the number of measurements made on each arm. The regression model suggests that the variance of error terms depends on the share randomized into each arm. However, only one randomization share per trial — namely the share not given a non-healing or lower class control - is measured. Therefore group-wise heteroskedasticity is permitted at the trial-level, but not at the arm-level. No further structure is imposed on the variance-covariance matrix. Only estimates where the dependent variable is calculated assuming subjects who attrite out heal at the same rate as those who are evaluated are reported. Results from regressions which assume that those who attrite out either all heal or all do not heal are not materially different.

Four specifications of $\bar{x}_{j}$ are estimated: (1) includes a constant and $d_{j}$; (2) adds trial-level variables (antacid usage, daily frequency of medication, total daily dosage of medication, total daily dosage of the more common drugs in the relevant class of medications) and their interactions with $d_{j}$; (3) adds subject-level variables (sex, smoker, and age) and their interactions with $d_{j}$; and (4) removes the trial-level variables and their interactions with $d_{j}$ from (3). Each specification is checked against Pregibon's link test. Those that fail are marked with a dagger $(\dagger)$. The residuals were checked for but did not reveal troubling patterns.

The first four columns of table 3 present results for treatment arms and different specifications of $\bar{x}_{j}$; the last four do the same for control arms. The first panel of the table gives the number of studies, arms, measurements on arms, and subjects included in each regression sample. The basic unit of observation is a measurement on an arm. (Although a number of observations are dropped because the survival rate is zero, the results are unchanged if survival rates of, e.g., 0.01, are 
substituted for survival rates of zero.) Although coefficient estimates are omitted, the second panel provides F-tests of the joint significance of certain subsets of regressors interacted with $d_{j}$. Their purpose is to test whether placebo effects operate through any of these subsets of variables. The main parameter of interest in this paper is the marginal effect of share treated on the daily hazard rate into the health state. Because the estimate for this marginal $-\partial\left[-\ln \left(\bar{y}_{j k t}\right) / t\right] / \partial d_{j}=\hat{\theta}_{(x d) k} \bar{x}_{j}$ - depends on the value of covariates, the third panel gives the estimated marginal effect (and standard deviation) at the mean value of the covariates in each regression. To provide a more complete picture of the distribution of the estimated marginal, the fourth panel gives the percentage of measurements where the share treated is estimated to have raised outcomes at different levels of confidence (employing a one-sided test). The fifth gives the percentage of measurements where the share treated either raised or depressed outcomes (employing a two-sided test).

The estimated marginal effect of share treated on outcomes at the mean value of covariates is positive in treatment arms across all specifications. All measurements in the first specification manifest a positive relationship at the $95 \%$ confidence level. One-half to two-thirds of measurements in other specifications manifest a positive relationship at the $95 \%$ level. In contrast, the estimated marginal effect of share treated on outcomes at the mean value of covariates is negative in control arms. Indeed, between two-fifths and all measurements manifest a negative relationship significant at the $95 \%$ confidence level. Importantly, the F-tests of the effects of share treated and various covariates interacted with the share treated are statistically significant in both treatment and control arms across all specification.

If the covariates of a specification are able to control for selection, these findings suggest that the treatment arms of $\mathrm{H}_{2}$-blocker trials manifest significant evidence of placebo effects, but the control arms do not. If the covariates do not fully control for selection, then both arms of these trials manifest significant evidence of placebo effects. The treatment arms do so because they manifest a positive relationship between share treated and outcomes; the control arms do so because they manifest a non-zero relationship. The results of the F-tests do not permit one to conclude that placebo effects fail to operate through any natural subset of variables.

Table 4 presents results for the remaining classes of anti-ulcer drugs, though F-tests are omitted. Arms treated with prostaglandins manifest weak evidence of placebo effects. While no arms manifest evidence of a positive relationship, nearly all manifest a non-zero relationship at the $95 \%$ 
confidence level under specifications one and two. However, the sample size of these regressions is rather small. Arms treated with proton-pump inhibitors manifest moderate evidence of placebo effects. Around a quarter of measurements manifest a significant positive relationship in specifications two to four. At least a quarter, and often nearly all, measurements manifest evidence of a non-zero relationship at the $90 \%$ confidence level.

Turning to the control arms of these trials, one finds moderate evidence of placebo effects in the control arms of prostaglandin trials. Over one-half of measurements manifest a positive relationship in specifications three and four. Around a third of measurements on control arms of proton-pump inhibitor trials manifest evidence of a positive relationship under specifications three and four. Around three-quarters of measurements manifest evidence of a significant non-zero relationship in specifications two through four. (There appears to be stronger evidence of placebo effects in specifications 3 and 4 for each drug and arm, perhaps because subject-level covariates do a better job of picking up the selection effects, permitting $d_{j}$ to focus on placebo effects.)

Only trials where published reports confirm that subjects were asked for informed consent were included in these regressions. The sample also contains, however, 28 trials of $\mathrm{H}_{2}$-blockers where it cannot be confirmed that informed consent was requested. Regressions employing only these trials reveal diminished evidence of placebo effects in specification one and two. The remaining specifications cannot be estimated because of the small sample size. (Results omitted.) This finding is consistent with the assumption that individuals learn the probability of receiving the experimental treatment via informed consent. Without this disclosure, an individual's beliefs about the probability of treatment are unrelated to the actual share treated. Therefore, outcomes, even if they depend on beliefs, should be invariant to the share treated in a trial.

\section{CONCLUSION}

This paper provides evidence of placebo effects in blinded, parallel-arm RCTs of $\mathrm{H}_{2}$-blockers and proton-pump inhibitors. This finding has important clinical implications. Placebo effects undermine the internal validity of clinical trials by causing investigators to underestimate the effect of treatment on the treated. Outside the context of trials, enrollees would know whether they are taking medication. Inside a blinded trial, they do not. Those randomized into the experimental- 
treatment group underperform — relative to those taking the same treatment outside the trial — because they think there is a chance they are getting the less valuable control treatment. Conversely, those randomized into the control group overperform because there is chance they may be getting the more valuable experimental treatment. The difference in mean outcomes across the two groups will therefore be less than the difference in outcomes outside the trial.

The size of this bias may be significant. Using table 2, the difference-in-means estimator suggests that the physiological effect of $\mathrm{H}_{2}$-blockers is a $2.4 \%$ increase in the daily healing rate. The size of placebo effects and thus the extent of bias due to their omission from estimates of treatment effects can be approximated by the estimated marginal effect of share treated on outcomes. Specification two in table 3 suggests that the estimated marginal may be as large as a $1.2 \%$ increase in the daily health rate. In other words, placebo effects may be $50 \%$ as large as the physiological effect of $\mathrm{H}_{2}$-blockers and that standard estimates of the treatment effect of these drugs is one-third smaller than they should be.

Nevertheless, further investigation is required before placebo effects can be labeled a serious and general medical phenomenon. This research should proceed in two directions. One is theoretical. This paper assumes health outcomes follow a simple linear model and examines only one type of trial. It would be useful to have a more general test for placebo effects that relaxes these restrictions. A second direction for research is application to other medical drugs and ailments. This is necessary to dispel concern that evidence of placebo effects in this paper are an artifact of data from ulcer trials.

If placebo effect are found to be a widespread phenomenon, two questions will naturally follow. First, why do beliefs affect outcomes? One theory is that placebo effect are a physiological phenomenon. Perhaps knowledge of a higher probability of treatment triggered greater production of mucous lining in subjects. An alternative theory is that placebo effects are behavioral. Perhaps knowledge of a higher probability of treatment caused subjects to take greater care of themselves by, e.g., reducing their consumption of caffeine. Because investigators did not observe this, it appears as if the share treated directly raised outcomes. Although both explanations imply that investigators underestimate treatment effects, this paper does not provide enough information to discriminate between these theories.

Second, can placebo effects be used for therapeutic purposes? For example, can a doctor cure 
a patient by suggesting, e.g., that a drug is more effective than it really is? If so, and if fooling patients is less costly than producing drugs, then placebo effects may be able to reduce the costs of health care. The existence of rational expectations may limit the productivity of placebo effects as a long-term cost-cutting measure. But the short-run cost savings might be non-trivial.

\section{A PROOFS}

Proposition 3. All expectations are taken with respect to $\mathbf{g}_{\pi}$. Independence of $\tilde{\pi}_{k i}$ and $\pi_{0 i}$ for $k=1,2$ implies that $\partial E\left[\pi_{1 i} \mid \tilde{\pi}_{1 i}>\tilde{\pi}_{2 i} / d\right] / \partial d=0$ and $\partial E\left[\pi_{1 i} \mid \tilde{\pi}_{1 i}>\tilde{\pi}_{2 i} / d\right] / \partial d=\partial E\left[\tilde{\pi}_{1 i} \mid \tilde{\pi}_{1 i}>\right.$ $\left.\tilde{\pi}_{2 i} / d\right] / \partial d$. Given $\tilde{\pi}_{k i}>0$, for $k=1,2, E\left[\tilde{\pi}_{1 i} \mid \tilde{\pi}_{1 i}>\tilde{\pi}_{2 i} / d\right]=E\left[\tilde{\pi}_{1 i} \mid \ln \tilde{\pi}_{1 i}-\ln \tilde{\pi}_{2 i}>-\ln d\right]$. Because $\ln x$ is monotone increasing in $x$,

$$
\operatorname{sign}\left(\frac{\partial E\left[\tilde{\pi}_{1 i} \mid \ln \tilde{\pi}_{1 i}-\ln \tilde{\pi}_{2 i}>-\ln d\right]}{\partial d}\right)=\operatorname{sign}\left(\frac{\partial E\left[\ln \tilde{\pi}_{1 i} \mid \ln \tilde{\pi}_{1 i}-\ln \tilde{\pi}_{2 i}>-\ln d\right]}{\partial d}\right) .
$$

Because $W_{i}$ and $V_{i}$ are independent, $E\left[\ln \tilde{\pi}_{1 i} \mid \ln \tilde{\pi}_{1 i}-\ln \tilde{\pi}_{2 i}>-\ln d\right]=\mu_{1}+b_{1} E\left(W_{i} \mid W_{i}>c(d, \mu)\right)$, where $c(d, \mu)=-\left(\mu_{1}-\mu_{2}\right)-\ln d$.

Log-concavity or log-convexity of $\left(\ln \tilde{\pi}_{1 i}, \ln \tilde{\pi}_{2 i}\right)$ implies log-concavity or log-convexity, respectively, of $W_{i}$, by corollary 2 in An (An, 1998). Propositions 1 and 2 in Heckman and Honore (Heckman and Honore, 1990) demonstrate that log-concavity or log-convexity of $W_{i}$ implies $\partial E\left[W_{i} \mid W_{i} \geq c\right] / \partial c \geq 0 . \quad$ Therefore,

$$
\frac{\partial E\left[\ln \tilde{\pi}_{1 i} \mid \ln \tilde{\pi}_{1 i}-\ln \tilde{\pi}_{2 i}>-\ln d\right]}{\partial d}=\frac{\sigma_{11}-\sigma_{12}}{\sigma} \frac{\partial E\left[W_{i} \mid W_{i} \geq c\right]}{\partial c} \frac{\partial c(d, \mu)}{\partial d} .
$$

Because $\partial c(d, \mu) / \partial d<0, \partial E\left[\ln \tilde{\pi}_{1 i} \mid \ln \tilde{\pi}_{1 i}-\ln \tilde{\pi}_{2 i}>-\ln d\right] / \partial d \leq 0$ so long as $\sigma_{11}>\sigma_{12}$. This condition is the same as $\rho_{12}<\sigma_{1} / \sigma_{2}$. Truncation of the range of $\tilde{\pi}_{k i}$ at, e.g., one does not alter this result (An, 1996).

Proposition 4. All expectations are taken with respect to $\mathbf{g}_{\pi}$. By (1)

$$
\begin{aligned}
& \frac{\partial E\left[y_{k i} \mid \tilde{\pi}_{1 i}>\tilde{\pi}_{2 i} / d\right]}{\partial d}=\left(1-a_{k}\right) \frac{\partial E\left[p_{k i} \mid \tilde{\pi}_{1 i}>\tilde{\pi}_{2 i} / d\right]}{\partial d} \\
& +a_{k}\left[d \frac{\partial E\left[\tilde{\pi}_{1 i} \mid \tilde{\pi}_{1 i}>\tilde{\pi}_{2 i} / d\right]}{\partial d}+\frac{\partial E\left[\pi_{0 i} \mid \tilde{\pi}_{1 i}>\tilde{\pi}_{2 i} / d\right]}{\partial d}\right]+a \tilde{\pi}_{1 i} .
\end{aligned}
$$


Working backwards, the selection equation (2) implies $\tilde{\pi}_{1 i}>0$. By assumption, $\partial E\left[\pi_{0 i} \mid \tilde{\pi}_{1 i}>\right.$ $\left.\tilde{\pi}_{2 i} / d\right] / \partial d=0 . \quad$ The proof to Proposition 3 demonstrates that $\operatorname{sign}\left(\partial E\left[\tilde{\pi}_{1 i} \mid \tilde{\pi}_{1 i}>\tilde{\pi}_{2 i} / d\right] / \partial d\right)=$ $\operatorname{sign}\left(\partial E\left[\pi_{1 i} \mid \tilde{\pi}_{1 i}>\tilde{\pi}_{2 i} / d\right] / \partial d\right)$, so $\partial E\left[\tilde{\pi}_{1 i} \mid \tilde{\pi}_{1 i}>\tilde{\pi}_{2 i} / d\right] / \partial d \leq 0$. By $(3)$,

$$
\frac{\partial E\left[p_{k i} \mid \tilde{\pi}_{1 i}>\tilde{\pi}_{2 i} / d\right]}{\partial d}=\frac{\partial E\left[f\left(\pi_{k i}\right) \mid \tilde{\pi}_{1 i}>\tilde{\pi}_{2 i} / d\right]}{\partial d}+\frac{\partial E\left[v_{k i} \mid \tilde{\pi}_{1 i}>\tilde{\pi}_{2 i} / d\right]}{\partial d}
$$

The last term is zero because $v_{k i}$ is assumed independent of $\pi_{k^{\prime} i^{\prime}}$ for all $\left(k^{\prime}, i^{\prime}\right)$ and of $v_{k^{\prime} i^{\prime}}$ for all $\left(k^{\prime}, i^{\prime}\right)$ except $\left(k^{\prime}=k, i^{\prime}=i\right)$. Since $f^{\prime}>0, \operatorname{sign}\left(\partial E\left[f\left(\pi_{k i}\right) \mid \tilde{\pi}_{1 i}>\tilde{\pi}_{2 i} / d\right]\right)=\operatorname{sign}\left(\partial E\left[\pi_{k i} \mid \tilde{\pi}_{1 i}>\right.\right.$ $\left.\left.\tilde{\pi}_{2 i} / d\right]\right)$. So $\partial E\left[p_{1 i} \mid \tilde{\pi}_{1 i}>\tilde{\pi}_{2 i} / d\right] / \partial d \leq 0$ and $\partial E\left[p_{0 i} \mid \tilde{\pi}_{1 i}>\tilde{\pi}_{2 i} / d\right] / \partial d=0$. If there are no placebo effects, i.e., $a_{k}=0$, then $\partial E\left[y_{1 i} \mid \tilde{\pi}_{1 i}>\tilde{\pi}_{2 i} / d\right] / \partial d \leq 0$ and $\partial E\left[y_{0 i} \mid \tilde{\pi}_{1 i}>\tilde{\pi}_{2 i} / d\right] / \partial d=0$. If, however, there exist placebo effects, the sign of $\partial E\left[y_{k i} \mid \tilde{\pi}_{1 i}>\tilde{\pi}_{2 i} / d\right] / \partial d$ is ambiguous.

\section{References}

An, M. Y. (1996), "Log-concave Probability Distributions: Theory and Statistical Testing," Centre for Labour Market and Social Research, University of Aarhus, Working Paper 96-01.

— (1998), "Logconcavity versus Logconvexity: A Complete Characterization," Journal of Economic Theory, 80, 350-369.

Heckman, J. and Honore, B. E. (1990), "The Empirical Content of the Roy Model," Econometrica, 58, 1121-1149.

Hrobjartsson, A. and Gotzsche, P. C. (2001), "Is the Placebo Powerless? An Analysis of Clinical Trials Comparing Placebo with No Treatment," New England Journal of Medicine, 344, 15941602.

Jensen, M. P. and Karoly, P. (1991), "Motivation and Expectancy Factors in Symptom Perception: A Laboratory Study of the Placebo Effect," Psychosomatic Medicine, 53, 144-152.

Kirsch, I. and Sapirstein, G. (1998), "Listening to Prozac but Hearing Placebo: A Meta-Analysis of Antidepressant Medication," Prevention and Treatment, 1, http://www.journals.apa.org/prevention/ volume1/pre0010002a.html. 
Kirsch, I. and Weixel, L. J. (1988), "Deouble-Blind Versus Deceptive Administration of a Placebo," Behavioral Neuroscience, 102, 319-323.

Kirsch, Irving, T. J. M. A. S. and Nicholls, S. S. (2002), "The Emperor's New Drugs: An Analysis of Antidepressant Medication Data Submitted to the U.S. Food and Drug Administration," Prevention and Treatment, 5, http://www.journals.apa.org/prevention/ volume5/pre0050023a.html.

Marlatt, G. A. and Rohsenow, D. J. (1980), "Cognitive processes in alcohol use: expectancy and the balanced placebo design," in Advances in Substance Abuse, ed. Mello, N., Greenwich, Conn.: JAI Press.

Penick, S. and Hinkle, L. E. (1964), "The Effect of Expectation on Response to Phenmetrazine," Psychosomatic Medicine, 26, 369-374.

Penick, S. B. and Fisher, S. (1965), "Drug-Set Interaction: Psychological and Physiological Effects of Epiephrine Under Differential Expectations," Psychosomatic Medicine, 27, 177-182.

Philipson, T. and DeSimone, J. (1997), "Experiments and Subject Sampling," Biometrika, 84, $221-234$.

Pollo, Antonella, M. A. A. A. C. C. G. M. and Benedetti, F. (2001), "Response expectancies in placebo analgesia and their clinical relevance," Pain, 93, 77-84.

Pregibon, D. (1980), "Goodness of link tests for generalized linear models," Applied Statistics, 29, $15-24$.

Price, Donald D., L. S. M. I. K. A. D. G. H. M. and Nicholls, S. S. (1999), "An analysis of factors that contribute to the magnitude of placebo analgesia in an experimental paradigm," Pain, 83, $147-156$.

Savage, L. J. (1954), The Foundations of Statistics, New York: John Wiley and Sons.

Skovlund, E. (1991), "Should we tell trial patients that they might receive placebo?" Lancet, 337, 1041. 
Table 1: Summary of tests for placebo effects.

\begin{tabular}{|l|c|c|c|c|c|}
\hline \multicolumn{2}{|l|}{ Conditions } & \multicolumn{2}{c|}{$\begin{array}{c}\text { Prediction for } \\
\partial E\left[y_{k} \mid B T\right] / \partial d\end{array}$} \\
\hline $\begin{array}{l}\text { Self- } \\
\text { selection }\end{array}$ & $\begin{array}{c}\text { Corr } \\
\left(\pi_{k}, p_{k}\right)\end{array}$ & $\begin{array}{c}\text { Consider } \\
\text { plac. effect }\end{array}$ & Other & $\begin{array}{c}\text { Treatment } \\
\text { group }\end{array}$ & $\begin{array}{c}\text { Control } \\
\text { group }\end{array}$ \\
\hline No & $\cdot$ & $\cdot$ & $\cdot$ & + & + \\
\hline Yes & 0 & $\cdot$ & $\cdot$ & $+/-$ & $+/-$ \\
\hline Yes & + & No & $\begin{array}{l}\tilde{\pi}_{k}, \pi_{0} \text { indep.; } ; \text { log concave } \\
\text { or convex; } W_{i}, V_{i} \text { uncorrel. }\end{array}$ & + & $+/-$ \\
\hline Yes & + & Yes & Same; $d>0.5$ & + & $+/-$ \\
\hline
\end{tabular}

Table 2: Summary statistics on data from ulcer trials, by drug type and arm.

\begin{tabular}{|c|c|c|c|c|c|c|c|c|c|}
\hline \multirow[t]{2}{*}{ Treatment } & \multicolumn{3}{|c|}{ "H2-Blockers } & \multicolumn{3}{|c|}{$\begin{array}{l}\text { Prostaglandins } \\
\end{array}$} & \multicolumn{3}{|l|}{ PPIs } \\
\hline & Obs. & Mean & SD & Obs. & Mean & SD & Obs. & Mean & SD \\
\hline \multicolumn{10}{|l|}{ Treatment arm } \\
\hline Share of subjects not given control & 266 & 0.88 & 0.20 & 46 & 0.56 & 0.08 & 97 & 0.61 & 0.16 \\
\hline Share of arms in placebo-control trials & 266 & 0.21 & 0.41 & 46 & 0.47 & 0.50 & 97 & 0.08 & 0.27 \\
\hline Share in antacid-control trials & 266 & 0.05 & 0.22 & 46 & 0.00 & 0.00 & 97 & 0.00 & 0.00 \\
\hline Share in lower-class drug control trials & 266 & 0.02 & 0.16 & 46 & 0.53 & 0.50 & 97 & 0.84 & 0.36 \\
\hline Share in same-class drug control trials & 266 & 0.72 & 0.45 & 46 & 0.00 & 0.00 & 97 & 0.08 & 0.27 \\
\hline Number enrolled & 266 & 186 & 144 & 46 & 123 & 110 & 97 & 110 & 40 \\
\hline Number evaluated (per protocol) & 266 & 170 & 130 & 46 & 108 & 96 & 97 & 102 & 37 \\
\hline Share of subjs. not healed (method 1) & 266 & 0.23 & 0.17 & 46 & 0.36 & 0.18 & 97 & 0.22 & 0.18 \\
\hline Daily hazard rate (meth. 1) & 253 & 0.049 & 0.014 & 46 & 0.036 & 0.018 & 82 & 0.087 & 0.029 \\
\hline Antacids permitted in trial $(1-5) ?$ & 266 & 3.6 & 0.7 & 46 & 3.5 & 0.6 & 97 & 3.1 & 1.2 \\
\hline Frequency of dosage (times/day) & 266 & 1.8 & 1.0 & 46 & 2.8 & 1.0 & 97 & 1.2 & 0.7 \\
\hline Total daily dosage (mg) & 266 & 0.440 & 0.358 & 46 & 0.093 & 0.233 & 97 & 0.028 & 0.018 \\
\hline Share male & 252 & 0.72 & 0.07 & 41 & 0.73 & 0.09 & 95 & 0.70 & 0.09 \\
\hline Share that smoke & 221 & 0.60 & 0.43 & 43 & 0.54 & 0.13 & 91 & 0.49 & 0.11 \\
\hline Average age (years) of subjs. & 247 & 46 & 4 & 41 & 44 & 5 & 87 & 46 & 5 \\
\hline \multicolumn{10}{|l|}{ Control arm } \\
\hline Share of subjects $n$ & 94 & 0.56 & 0.11 & 40 & 0.53 & 0.07 & 67 & 0.53 & 0.08 \\
\hline Share of arms in placebo-control trials & 94 & 0.77 & 0.43 & 40 & 0.39 & 0.49 & 67 & 0.04 & 0.19 \\
\hline Share in antacid-control trials & 94 & 0.30 & 0.46 & 40 & 0.00 & 0.00 & 67 & 0.00 & 0.00 \\
\hline Share in lower-class drug control trials & 94 & 0.00 & 0.00 & 40 & 0.61 & 0.49 & 67 & 0.96 & 0.19 \\
\hline Share in same-class drug control trials & 94 & 0.00 & 0.00 & 40 & 0.00 & 0.00 & 67 & 0.00 & 0.00 \\
\hline Number enrolled & 94 & 86 & 84 & 40 & 130 & 116 & 67 & 113 & 39 \\
\hline Number evaluated (per protocol) & 94 & 76 & 75 & 40 & 117 & 105 & 67 & 104 & 38 \\
\hline Share of subjs. not healed (meth. 1) & 94 & 0.57 & 0.23 & 39 & 0.36 & 0.28 & 67 & 0.36 & 0.22 \\
\hline Daily hazard rate (meth. 1) & 93 & 0.025 & 0.017 & 38 & 0.040 & 0.021 & 66 & 0.055 & 0.021 \\
\hline Antacids permitted in trial (1-5)? & 94 & 3.3 & 1.0 & 40 & 3.5 & 0.6 & 67 & 3.2 & 1.2 \\
\hline Share male & 85 & 0.76 & 0.10 & 35 & 0.77 & 0.09 & 65 & 0.70 & 0.09 \\
\hline Share that smoke & 67 & 0.64 & 0.12 & 37 & 0.52 & 0.11 & 61 & 0.49 & 0.14 \\
\hline Average age (years) of subjs. & 76 & 45 & 5 & 35 & 44 & 5 & 59 & 46 & 6 \\
\hline
\end{tabular}


Table 3: Evidence of placebo effects from H2-blocker trials.

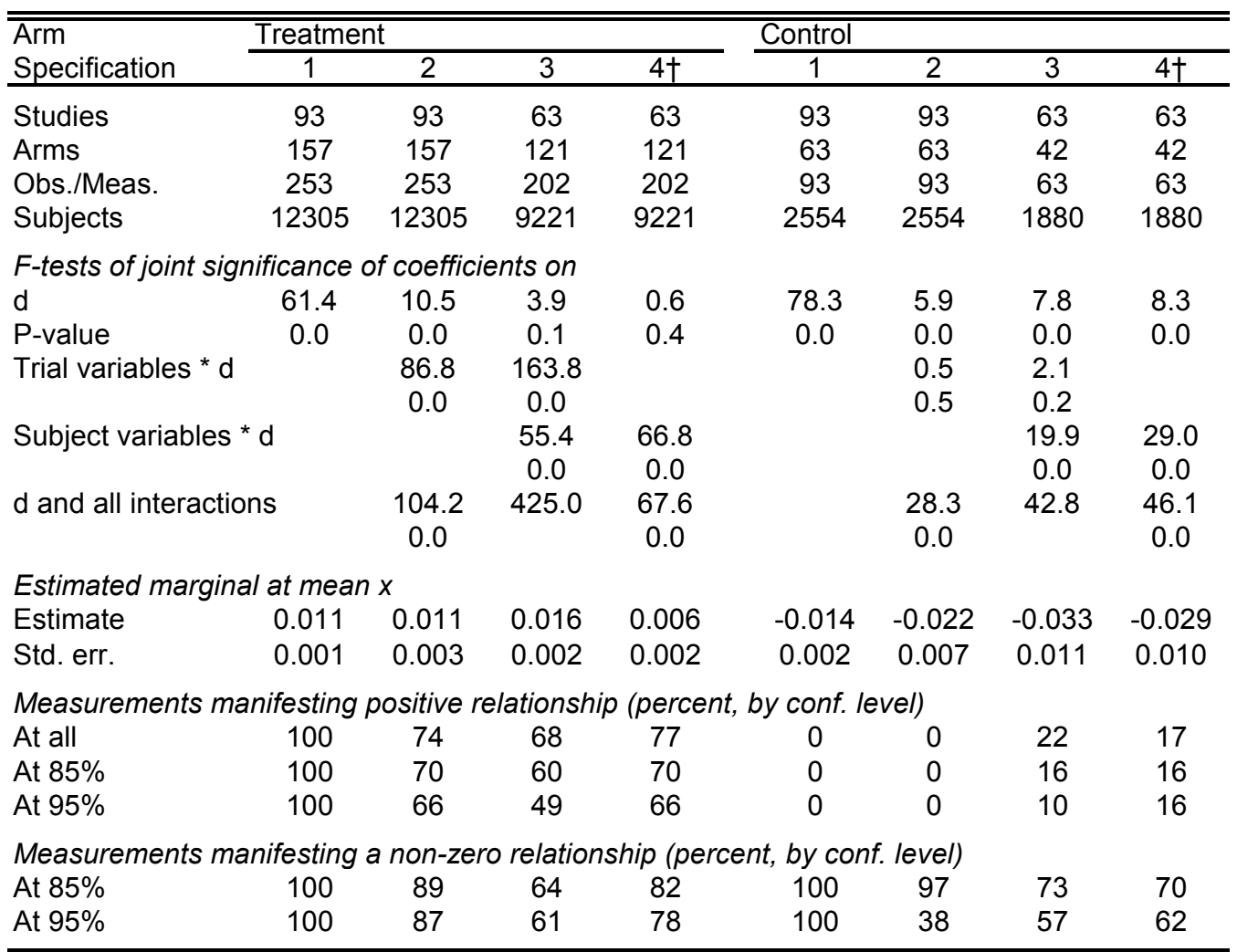


Table 4: Evidence of placebo effects in prostaglandin and PPI trials.

\begin{tabular}{|c|c|c|c|c|c|c|c|c|}
\hline \multirow{2}{*}{$\begin{array}{l}\text { Treatment } \\
\text { Specification }\end{array}$} & \multicolumn{4}{|c|}{ Prostagladin } & \multicolumn{4}{|l|}{$\overline{\mathrm{PPI}}$} \\
\hline & 1 & 2 & 3 & 4 & 1 & 2 & 3 & 4 \\
\hline \multicolumn{9}{|c|}{ Treatment arms } \\
\hline Studies & 38 & 38 & 33 & 33 & 66 & 66 & 56 & 56 \\
\hline Obs./meas. & 46 & 46 & 46 & 46 & 82 & 82 & 70 & 70 \\
\hline Subjects & 1497 & 1497 & 1497 & 1497 & 3547 & 3547 & 2977 & 2977 \\
\hline \multicolumn{9}{|c|}{ Estimated marginal effect of share treated on the daily hazard (at mean $x$ ) } \\
\hline Estimate & -0.038 & -28.3 & -0.038 & -0.038 & -0.022 & 0.005 & -0.062 & -0.030 \\
\hline Std. err. & 0.012 & 3.927 & 0.012 & 0.012 & 0.013 & 0.015 & 0.026 & 0.013 \\
\hline \multicolumn{9}{|c|}{ Measurements manifesting positive relationship (percent, by conf. level) } \\
\hline At $90 \%$ & 0 & 0 & 0 & 0 & 0 & 20 & 30 & 36 \\
\hline At $95 \%$ & 0 & 0 & 0 & 0 & 0 & 20 & 30 & 36 \\
\hline \multicolumn{9}{|c|}{ Measurements manifesting a non-zero relationship (percent, by conf. level) } \\
\hline At $90 \%$ & 100 & 87 & 100 & 100 & 100 & 29 & 64 & 84 \\
\hline At $95 \%$ & 100 & 87 & 100 & 100 & 0 & 22 & 64 & 84 \\
\hline Control arms & & $\dagger$ & & & & & & \\
\hline$\overline{\text { Obs./meas. }}$ & 38 & 38 & 33 & 33 & 66 & 66 & 56 & 56 \\
\hline Subjects & 1171 & 1171 & 1069 & 1069 & 2686 & 2686 & 2233 & 2233 \\
\hline \multicolumn{9}{|c|}{ Estimated marginal effect of share treated on the daily hazard (at mean $x$ ) } \\
\hline Estimate & -0.020 & -0.082 & 0.262 & 0.389 & 0.014 & 0.01 & -0.02 & -0.02 \\
\hline Std. err. & 0.043 & 0.039 & 0.098 & 0.126 & 0 & 0.08 & 0.28 & 0.29 \\
\hline \multicolumn{9}{|c|}{ Measurements manifesting positive relationship (percent, by conf. level) } \\
\hline At $90 \%$ & 0 & 0 & 61 & 64 & 0 & 15 & 36 & 39 \\
\hline At $95 \%$ & 0 & 0 & 61 & 64 & 0 & 15 & 32 & 32 \\
\hline \multicolumn{9}{|c|}{ Measurements manifesting a non-zero relationship (percent, by conf. level) } \\
\hline At $90 \%$ & 0 & 79 & 73 & 82 & 0 & 79 & 75 & 75 \\
\hline At $95 \%$ & 0 & 79 & 58 & 82 & 0 & 79 & 70 & 75 \\
\hline
\end{tabular}

